Article

\title{
Dynamic Analysis of a Particle Motion System
}

\author{
Ning Cui ${ }^{1, *(1)}$ and Junhong $\mathrm{Li}^{1,2}$ \\ 1 Department of Mathematics and Sciences, Hebei Institute of Architecture and Civil Engineering, \\ Zhangiiakou 075000, Hebei Province, China; jhli2011@163.com \\ 2 School of Mathematics and Statistics, Beijing Institute of Technology, Beijing 100081, China \\ * Correspondence: cnmath80@163.com
}

Received: 22 November 2018; Accepted: 16 December 2018; Published: 21 December 2018

\begin{abstract}
This paper formulates a new particle motion system. The dynamic behaviors of the system are studied including the continuous dependence on initial conditions of the system's solution, the equilibrium stability, Hopf bifurcation at the equilibrium point, etc. This shows the rich dynamic behaviors of the system, including the supercritical Hopf bifurcations, subcritical Hopf bifurcations, and chaotic attractors. Numerical simulations are carried out to verify theoretical analyses and to exhibit the rich dynamic behaviors.
\end{abstract}

Keywords: particle motion; Hopf bifurcation; chaos

MSC: 34C23; 34C28

\section{Introduction}

There are some scholars who have studied the dynamic behaviors of particle motion. The results show that particle motion is a complex dynamic behavior in some case, such as chaotic motion. For instance, Abbott N. L. investigated the diffusion of a colloidal particle in a liquid crystalline solvent [1]. Chen C. and his colleague studied the chaotic particle dynamics in free-electron lasers and obtained that the particle motion becomes chaotic on a time scale. Here, the time scale is the characteristic time scale for radial-gradient-induced changes in the particle orbits, which is shown to be of the order of the beam transit time through a few wiggler periods [2]. Research showed that the chaos of a particle probing the black hole horizon had a universal upper bound for the Lyapunov exponent [3]. Since chaos began to be studied, it has been a common belief that understanding and utilizing the rich dynamics of a nonlinear system have an important impact on modern technology. Therefore, it also promotes the study of chaos, and some useful results have been obtained. For example, Sprott J. C. and Xiong A. [4] presented a method for classifying basins of attraction and quantifying their size for any dissipative dynamical system, and the results were useful to describe the basin of attraction and quantifying its shape and size for both theoretical and practical reasons. By using the Pynamical software package, Boeing G. [5] investigated visualization methods of nonlinear dynamical systems' behavior and indicated that these methods can help researchers discover, examine, and understand the behaviors of nonlinear dynamical systems, including bifurcations, the path to chaos, fractals, and strange attractors. Bradley E. and Kantz H. [6] illustrated that the results of nonlinear time-series analysis can be helpful in understanding, characterizing, and predicting dynamical systems. In fact, chaos has many manifestations in many different situations [7]. Meanwhile, many systems will appear with multiple equilibrium points under some parameter conditions, and the increase of the equilibrium points or multi-equilibrium points may lead to richer dynamic behaviors of the system [8-10]. For these reasons, we will formulate a particle motion model under external force and discuss the Hopf bifurcation and chaotic behaviors of the system. 
In this paper, we will formulate a new model for particle motion and the stability of equilibrium points. The continuous dependence on initial conditions of the system's solution and Hopf bifurcation are investigated in Section 2. To further study the complex dynamic behaviors of particle motion, simulations including Lyapunov exponents, Poincaré maps and phase portraits of the chaotic attractor for the system are given in Section 3. A summary of our results and further discussion are presented in Section 4.

\section{Model}

There are rich dynamic behaviors in some cases, such as in sheared suspensions [11], in creeping flow [12], and around a weakly-magnetized Schwarzschild black hole [13]. This shows that the particle motion becomes complex because of the existence of external force, and the particle system has different dynamic behaviors under different external forces [14-16]. Here, we assume that a particle with unit mass is moving on a horizontal smooth plane $(p, q)$, and the forces on the particle in $p$ and $q$ direction are $F_{p}$ and $F_{q}$, respectively, where:

$$
F_{p}=a_{11} p-a_{12} p^{3}+a_{13} \dot{q}-a_{14} \dot{p}, F_{q}=a_{11} q-a_{12} q^{3}+a_{13} p-a_{14} \dot{q} .
$$

$a_{11}, a_{12}, a_{13}$, and $a_{14}$ are all positive parameters. The dot expresses the derivative with respect to the time variable $t$. Then, the particle motion equations are described by:

$$
\left\{\begin{array}{l}
\dot{p}=u, \\
\dot{u}=a_{11} p-a_{12} p^{3}+a_{13} v-a_{14} u, \\
\dot{q}=v \\
\dot{v}=a_{11} q-a_{12} q^{3}+a_{13} p-a_{14} v .
\end{array}\right.
$$

\subsection{Symmetry and Dissipation}

Obviously, System (1) is symmetric with coordinate transformations:

$$
(p, u, q, v) \rightarrow(-p,-u,-q,-v) .
$$

The divergence of (1) is:

$$
\nabla V=\frac{\partial \dot{p}}{\partial p}+\frac{\partial \dot{u}}{\partial u}+\frac{\partial \dot{q}}{\partial q}+\frac{\partial \dot{v}}{\partial v}=-2 a_{14}<0 .
$$

Thus, the system is dissipative. This indicates that the volume element $V_{0} e^{-2 a_{14} t}$ as $t \rightarrow \infty$, then all the trajectories of the system (1) are ultimately in an attractor.

\subsection{Existence and Uniqueness of the Solution}

We first rewrite the system (1) as follows:

$$
\dot{P}(t)=Q(P(t)), t \in(0, T],
$$

where:

$$
P=\left[\begin{array}{c}
p \\
u \\
q \\
v
\end{array}\right], Q(P)=\left[\begin{array}{c}
u \\
a_{11} p-a_{12} p^{3}+a_{13} v-a_{14} u \\
v \\
a_{11} q-a_{12} q^{3}+a_{13} p-a_{14} v
\end{array}\right]
$$

Let

$$
D_{1}=\{(p, u, q, v): \max \{|p|,|u|,|q|,|v|\} \leq M\}
$$

and $M>0$. The existence and uniqueness of the solution are studied in the region $D_{1} \times D_{2}$ where $D_{2}=(0, T]$. The solution of (2) with initial conditions $P(0)=P_{0}$ is: 


$$
P=P_{0}+\int_{0}^{t} Q(P(\tau)) d \tau
$$

where:

$$
P_{0}=\left[\begin{array}{l}
p_{0} \\
u_{0} \\
q_{0} \\
v_{0}
\end{array}\right] .
$$

Next, we denote the right-hand side of (3) by $W(P)$, then:

$$
W\left(P_{1}\right)-W\left(P_{2}\right)=\int_{0}^{t}\left(Q\left(P_{1}(\tau)\right)-Q\left(P_{2}(\tau)\right)\right) d \tau,
$$

here:

$$
P_{1}=\left[\begin{array}{l}
p_{1} \\
u_{1} \\
q_{1} \\
v_{1}
\end{array}\right], P_{2}=\left[\begin{array}{l}
p_{2} \\
u_{2} \\
q_{2} \\
v_{2}
\end{array}\right]
$$

Therefore,

$$
\begin{aligned}
& W\left(P_{1}\right)-W\left(P_{2}\right) \leq \int_{0}^{t}\left|\left(Q\left(P_{1}(\tau)\right)-Q\left(P_{2}(\tau)\right)\right)\right| d \tau \\
\leq & \operatorname{Tmax}\left[\begin{array}{c}
\left|p_{1}-p_{2}\right| \\
a_{11}\left|p_{1}-p_{2}\right|+a_{12}\left|p_{1}^{3}-p_{2}^{3}\right|+a_{13}\left|v_{1}-v_{2}\right|+a_{14}\left|u_{1}-u_{2}\right| \\
\left|v_{1}-v_{2}\right| \\
a_{11}\left|q_{1}-q_{2}\right|+a_{12}\left|q_{1}^{3}-q_{2}^{3}\right|+a_{13}\left|p_{1}-p_{2}\right|+a_{14}\left|v_{1}-v_{2}\right|
\end{array}\right] .
\end{aligned}
$$

The supremum norm is defined for the class of continuous function $C[0, T]$ by:

$$
\|\Phi\|=\sup _{t \in(0, T]}|\Phi(t)|, \Phi(t) \in C[0, T],
$$

and for a matrix $A=\left[a_{i j}(t)\right]$, which is defined by $\|A\|=\sum_{i, j} \sup _{t \in(0, T]}\left|a_{i j}(t)\right|$. Thus, we obtain:

$$
\begin{gathered}
\left\|W\left(P_{1}\right)-W\left(P_{2}\right)\right\|=T \sup \left\{\left|p_{1}-p_{2}\right|+a_{11}\left|p_{1}-p_{2}\right|+a_{12}\left|p_{1}-p_{2}\right|\left|p_{1}^{2}+p_{1} p_{2}+p_{2}^{2}\right|+a_{13}\left|v_{1}-v_{2}\right|\right. \\
\left.+a_{14}\left|u_{1}-u_{2}\right|+\left|v_{1}-v_{2}\right|+a_{11}\left|q_{1}-q_{2}\right|+a_{12}\left|q_{1}-q_{2}\right|\left|q_{1}^{2}+q_{1} q_{2}+q_{2}^{2}\right|+a_{13}\left|p_{1}-p_{2}\right|+a_{14}\left|v_{1}-v_{2}\right|\right\} \\
\leq T \max \left\{a_{14}, a_{11}+3 a_{12} M^{2}, 1+a_{13}+a_{14}, 1+a_{11}+a_{12}+a_{13}+3 M^{2}\right\}\left\|P_{1}-P_{2}\right\| \\
\leq \rho\left\|P_{1}-P_{2}\right\|,
\end{gathered}
$$

where:

$$
\rho=T \max \left\{a_{14}, a_{11}+3 a_{12} M^{2}, 1+a_{13}+a_{14}, 1+a_{11}+a_{12}+a_{13}+3 M^{2}\right\}>0 .
$$

The following theorem is obtained.

Theorem 1. The sufficient condition for the existence and uniqueness of the solution of (1) with initial conditions $P(0)=P_{0}$ in the region $D_{1} \times D_{2}$ is $0<\rho<1$.

\subsection{Continuous Dependence on Initial Conditions}

Based on the results in Section 2.1, we have:

$$
P_{1}=P_{10}+\int_{0}^{t} Q\left(P_{1}(\tau)\right) d \tau, P_{2}=P_{20}+\int_{0}^{t} Q\left(P_{2}(\tau)\right) d \tau,
$$

where: 


$$
P_{10}=\left[\begin{array}{l}
p_{10} \\
u_{10} \\
q_{10} \\
v_{10}
\end{array}\right], P_{20}=\left[\begin{array}{l}
p_{20} \\
u_{20} \\
q_{20} \\
v_{20}
\end{array}\right] .
$$

$P_{10}$ and $P_{20}$ are all the initial conditions to (2) and $\left\|P_{10}-P_{20}\right\| \leq \varepsilon$. Under the condition of Theorem 1 , the following inequality is obtained:

$$
\left\|P_{1}-P_{2}\right\| \leq\left\|P_{10}-P_{20}\right\|+\int_{0}^{t}\left|\left(Q\left(P_{1}(\tau)\right)-Q\left(P_{2}(\tau)\right)\right)\right| d \tau \leq\left\|P_{10}-P_{20}\right\|+\rho\left\|P_{1}-P_{2}\right\|,
$$

hence $\left\|P_{1}-P_{2}\right\| \leq \frac{\varepsilon}{1-\rho}$. In conclusion, $\forall \sigma>0, \exists \varepsilon(\sigma)=(1-\rho) \sigma>0$ such that $\left\|P_{10}-P_{20}\right\| \leq \varepsilon$ implies that $\left\|P_{1}-P_{2}\right\| \leq \sigma$.

Theorem 2. There is continuous dependence on the initial conditions of the solution of (1) under the condition of Theorem 1.

\subsection{Equilibrium and Stability}

It is easy to visualize that (1) always has three equilibrium points, i.e.,

$$
e_{0}=(0,0,0,0), e_{1}=\left(0,0, \sqrt{\frac{a_{11}}{a_{12}}}, 0\right), e_{2}=\left(0,0,-\sqrt{\frac{a_{11}}{a_{12}}}, 0\right) .
$$

When $R_{0}=\frac{1}{3}\left(\frac{2 a_{11}}{3 a_{13}}\right)^{2} \leq 1$, (1) has equilibria as follows:

$$
e_{3}=\left(\sqrt{\frac{a_{11}}{a_{12}}}, 0, \frac{\sqrt[3]{\theta_{1}}}{6 a_{12}}+\frac{2 a_{11}}{\sqrt[3]{\theta_{1}}}, 0\right), e_{4}=\left(-\sqrt{\frac{a_{11}}{a_{12}}}, 0, \frac{\sqrt[3]{\theta_{2}}}{6 a_{12}}+\frac{2 a_{11}}{\sqrt[3]{\theta_{2}}}, 0\right),
$$

where:

$$
\theta_{1}=\left(108 a_{13} \sqrt{\frac{a_{11}}{a_{12}}}+12 \sqrt{\frac{a_{11}\left(1-R_{0}\right)}{a_{12} a_{13}{ }^{2}}}\right) a_{12}{ }^{2}, \theta_{2}=\left(-108 a_{13} \sqrt{\frac{a_{11}}{a_{12}}}+12 \sqrt{\frac{a_{11}\left(1-R_{0}\right)}{a_{12} a_{13}{ }^{2}}}\right) a_{12}{ }^{2} .
$$

By calculations, the characteristic equations at equilibrium points are obtained as follows:

$$
\begin{aligned}
& f_{e_{0}}(\lambda)=\lambda^{4}+2 \lambda^{3} a_{14}+\left(a_{14}^{2}-2 a_{11}\right) \lambda^{2}+\left(-2 a_{11} a_{14}-a_{13}^{2}\right) \lambda+a_{11}^{2} ， \\
& f_{e_{1}}(\lambda)=f_{e_{2}}(\lambda)=\lambda^{4}+2 \lambda^{3} a_{14}+\left(a_{14}{ }^{2}+a_{11}\right) \lambda^{2}+\left(a_{11} a_{14}-a_{13}{ }^{2}\right) \lambda-2 a_{11}{ }^{2} \text {, } \\
& f_{e_{3}}(\lambda)=\lambda^{4}+2 \lambda^{3} a_{14}+\theta_{12} \lambda^{2}+\theta_{11} \lambda+\theta_{10}, \\
& \theta_{12}=\frac{12 \theta_{1}^{2 / 3} a_{12} a_{14}{ }^{2}+\theta_{1}{ }^{4 / 3}+36 a_{11} a_{12} \theta_{1}^{2 / 3}+144 a_{11}{ }^{2} a_{12}{ }^{2}}{12 \theta_{1}^{2 / 3} a_{12}}, \\
& \theta_{11}=\frac{\theta_{1}^{4 / 3} a_{14}+36 a_{11} a_{12} \theta_{1}^{2 / 3} a_{14}-12 \theta_{1}^{2 / 3} a_{12} a_{13}{ }^{2}+144 a_{11}{ }^{2} a_{12}{ }^{2} a_{14}}{12 \theta_{1}^{2 / 3} a_{12}}, \\
& \theta_{10}=\frac{2 \theta_{1}^{4 / 3} a_{11}+24 \theta_{1}^{2 / 3} a_{11}{ }^{2} a_{12}+288 a_{11}{ }^{3} a_{12}{ }^{2}}{12 \theta_{1}^{2 / 3} a_{12}}, \\
& f_{e_{4}}(\lambda)=\lambda^{4}+2 \lambda^{3} a_{14}+\theta_{22} \lambda^{2}+\theta_{21} \lambda+\theta_{20}, \\
& \theta_{22}=\frac{12 \theta_{2}^{2 / 3} a_{12} a_{14}{ }^{2}+\theta_{2}{ }^{4 / 3}+36 a_{11} a_{12} \theta_{2}^{2 / 3}+144 a_{11}{ }^{2} a_{12}{ }^{2}}{12 \theta_{2}^{2 / 3} a_{12}}, \\
& \theta_{21}=\frac{\theta_{2}^{4 / 3} a_{14}+36 a_{11} a_{12} \theta_{2}{ }^{2 / 3} a_{14}-12 \theta_{2}{ }^{2 / 3} a_{12} a_{13}{ }^{2}+144 a_{11}{ }^{2} a_{12}{ }^{2} a_{14}}{12 \theta_{2}{ }^{2 / 3} a_{12}}, \\
& \theta_{20}=\frac{2 \theta_{2}{ }^{4 / 3} a_{11}+24 \theta_{2}{ }^{2 / 3} a_{11}{ }^{2} a_{12}+288 a_{11}{ }^{3} a_{12}{ }^{2}}{12 \theta_{2}{ }^{2 / 3} a_{12}} .
\end{aligned}
$$

Obviously, $f_{e_{0}}(\lambda), f_{e_{1}}(\lambda)$, and $f_{e_{2}}(\lambda)$ have roots with positive real parts, and $e_{0}, e_{1}$, and $e_{2}$ are unstable. In the following, we will discuss the bifurcations at the rest of the equilibrium points.

\subsection{Hopf Bifurcation}

In this subsection, the Hopf bifurcation of System (1) is investigated by using the theories in [10].

When $f_{e_{3}}(\lambda)$ has a pair of pure imaginary roots $\lambda_{1,2}= \pm \alpha i, f_{e_{3}}(\lambda)=0$ becomes:

$$
\left(\lambda^{2}+\alpha^{2}\right)\left(\lambda^{2}+\xi \lambda+\eta\right)=0,
$$


then we get:

$$
\left\{\begin{array}{l}
\xi=2 a_{14} \\
\alpha^{2}=\frac{\theta_{11}}{2 a_{14}} \\
\eta=\theta_{12}-\frac{\theta_{11}}{2 a_{14}} \\
\frac{\theta_{11}}{2 a_{14}}=\frac{2 a_{14} \theta_{10}}{2 \theta_{12} a_{14}-\theta_{11}} .
\end{array}\right.
$$

By calculations,

$$
\Delta_{0}=\xi^{2}-4 \eta=-\frac{\theta_{1}^{4 / 3} a_{14}+36 a_{11} a_{12} \theta_{1}^{2 / 3} a_{14}+12 \theta_{1}^{2 / 3} a_{12} a_{13}{ }^{2}+144 a_{11}^{2} a_{12}{ }^{2} a_{14}}{6 \theta_{1}^{2 / 3} a_{12}}<0 .
$$

Therefore, the eigenvalues of Jacobian matrix at $e_{3}$ are:

$$
\lambda_{1}=\alpha i, \lambda_{2}=-\alpha i, \lambda_{3}=-a_{14}+\frac{\sqrt{-\Delta_{0}} i}{2}, \lambda_{4}=-a_{14}-\frac{\sqrt{-\Delta_{0}} i}{2} .
$$

By straightforward computations, we obtain the eigenvectors with respect to $\lambda_{1}=\alpha i$, i.e.,

$$
\beta_{1}=m\left(\begin{array}{c}
\frac{a_{14} \alpha i-\alpha^{2}-M}{a_{13}} \\
-\frac{\alpha\left(\alpha^{2} i+M i+a_{14} \alpha\right)}{a_{13}} \\
1 \\
\alpha i
\end{array}\right),
$$

where:

$$
M=-3\left(\frac{\sqrt[3]{\theta_{1}}}{6 a_{12}}+\frac{2 a_{11}}{\sqrt[3]{\theta_{2}}}\right)^{2} a_{12}+a_{11}
$$

Let $m=1, R_{e}$ and $I_{e}$ denote the real and imaginary parts of $\beta_{1}$, then:

$$
R_{e}=\left(\begin{array}{c}
\frac{-\alpha^{2}-M}{a_{13}} \\
-\frac{a_{14} \alpha^{2}}{a_{13}} \\
1 \\
0
\end{array}\right), I_{e}=\left(\begin{array}{c}
\frac{a_{14} \alpha}{a_{13}} \\
-\frac{\alpha\left(\alpha^{2}+M\right)}{a_{13}} \\
0 \\
\alpha
\end{array}\right)
$$

For $\lambda_{3}=i \frac{\sqrt{-\Delta_{0}}}{2}-a_{14}$, the eigenvectors are obtained:

$$
\beta_{2}=\left(\begin{array}{c}
\beta_{21} i+\beta_{22} \\
\beta_{23} i+\beta_{24} \\
1 \\
\beta_{25} i-a_{14}
\end{array}\right)=\left(\begin{array}{c}
-\frac{a_{14} \sqrt{-\Delta_{0}}}{2 a_{13}} i-\frac{4 M-\Delta_{0}}{4 a_{13}} \\
\frac{\sqrt{-\Delta_{0}}\left(\Delta_{0}-4 M+4 a_{14}^{2}\right) i}{8 a_{13}}+\frac{2 M a_{14}-a_{14} \Delta_{0}}{2 a_{13}} \\
1 \\
\frac{\sqrt{-\Delta_{0}} i}{2}-a_{14}
\end{array}\right)
$$

Next, we use the following transformation:

$$
\left(\begin{array}{c}
p-\sqrt{\frac{a_{11}}{a_{12}}} \\
u \\
q-\frac{\sqrt[3]{\theta_{1}}}{6 a_{12}}-\frac{2 a_{11}}{\sqrt[3]{\theta_{1}}} \\
v
\end{array}\right)=\left(\begin{array}{cccc}
\frac{-\alpha^{2}-M}{a_{13}} & -\frac{a_{14} \alpha}{a_{13}} & \beta_{22} & -\beta_{21} \\
-\frac{a_{14} \alpha^{2}}{a_{13}} & \frac{\alpha\left(\alpha^{2}+M\right)}{a_{13}} & \beta_{24} & -\beta_{23} \\
1 & 0 & 1 & 0 \\
0 & -\alpha & -a_{14} & -\beta_{25}
\end{array}\right)\left(\begin{array}{l}
y_{1} \\
y_{2} \\
y_{3} \\
y_{4}
\end{array}\right)
$$

Then, the system (1) becomes: 


$$
\left\{\begin{array}{l}
\dot{y}_{1}=-\alpha y_{2}+F_{1}\left(y_{1}, y_{2}, y_{3}, y_{4}\right) \\
\dot{y}_{2}=\alpha y_{1}+F_{2}\left(y_{1}, y_{2}, y_{3}, y_{4}\right) \\
\dot{y}_{3}=-a_{14} y_{3}-\frac{\sqrt{-\Delta_{0}}}{2} y_{4}+F_{3}\left(y_{1}, y_{2}, y_{3}, y_{4}\right) \\
\dot{y}_{4}=\frac{\sqrt{-\Delta_{0}}}{2} y_{3}-a_{14} y_{4}+F_{4}\left(y_{1}, y_{2}, y_{3}, y_{4}\right)
\end{array}\right.
$$

where the items $F_{i}\left(y_{1}, y_{2}, y_{3}, y_{4}\right)(i=1,2,3,4)$ can be seen in Appendix A.

According to the center manifold theorem [17], there exists a center manifold for (1) as follows:

$$
\begin{gathered}
W^{c}(0)=\left\{\left(y_{1}, y_{2}, y_{3}, y_{4}\right) \in R^{4}\left|y_{3}=h_{3}\left(y_{1}, y_{2}\right), y_{4}=h_{4}\left(y_{1}, y_{2}\right),\right|\left(y_{1}, y_{2}\right) \mid<\rho, h_{3}(0,0)=0,\right. \\
\left.D h_{3}(0,0)=0, h_{4}(0,0)=0, D h_{4}(0,0)=0\right\}
\end{gathered}
$$

for sufficiently small $\rho$. We assume that:

$$
h_{3}\left(y_{1}, y_{2}\right)=h_{31} y_{1}^{3}+h_{32} y_{1}^{2} y_{2}+h_{33} y_{1} y_{2}^{2}+\ldots, h_{4}\left(y_{1}, y_{2}\right)=h_{41} y_{1}^{3}+h_{42} y_{1}^{2} y_{2}+h_{43} y_{1} y_{2}^{2}+\ldots,
$$

where the items $h_{3 j}, h_{4 j}(j=1,2,3)$ can be seen in Appendix A. Then:

$$
\dot{y}_{3}=\frac{\partial h_{3}}{\partial y_{1}} \dot{y}_{1}+\frac{\partial h_{3}}{\partial y_{2}} \dot{y}_{2}, \dot{y}_{4}=\frac{\partial h_{4}}{\partial y_{1}} \dot{y}_{1}+\frac{\partial h_{4}}{\partial y_{2}} \dot{y}_{2} \text {. }
$$

Therefore, it is obtained that:

$$
\left\{\begin{array}{l}
\dot{y}_{1}=-\alpha y_{2}+G_{1}\left(y_{1}, y_{2}\right) \\
\dot{y}_{2}=\alpha y_{1}+G_{2}\left(y_{1}, y_{2}\right)
\end{array}\right.
$$

where the items $G_{1}\left(y_{1}, y_{2}\right)$ and $G_{2}\left(y_{1}, y_{2}\right)$ can be seen in Appendix A.

Therefore, applying the method in [17], we can compute the first-order fine focus as follows:

$$
\begin{gathered}
\mu=\left\{\frac{\pi}{8 \alpha}\left(G_{1 y_{1} y_{1} y_{1}}+G_{1 y_{1} y_{2} y_{2}}+G_{2 y_{1} y_{1} y_{2}}+G_{2 y_{2} y_{2} y_{2}}\right)+\frac{\pi}{8 \alpha^{2}}\left[G_{1 y_{1} y_{2}}\left(G_{1 y_{1} y_{1}}+G_{1 y_{2} y_{2}}\right)-G_{2 y_{1} y_{2}}\left(G_{2 y_{1} y_{1}}\right.\right.\right. \\
\left.\left.\left.+G_{2 y_{2} y_{2}}\right)-G_{1 y_{1} y_{1}} G_{2 y_{1} y_{1}}+G_{1 y_{2} y_{2}} G_{2 y_{2} y_{2}}\right]\right\}\left.\right|_{\left(y_{1}, y_{2}\right)=(0,0)}=\frac{\pi \mu_{1}}{8 \alpha}+\frac{\pi \mu_{2}}{8 \alpha^{2}}
\end{gathered}
$$

where the items $\mu_{1}$ and $\mu_{2}$ can be seen in Appendix A.

Theorem 3. The supercritical Hopf bifurcation of the system (1) occurs if $\mu<0$, and the subcritical Hopf bifurcation occurs if $\mu>0$.

The same approach can be used to study the Hopf bifurcation at the other equilibrium $e_{4}$.

\section{Simulation}

In this section, the example of Hopf bifurcations for the system is given, and it shows that chaotic phenomena occur in the system with some parameter values. Firstly, we fix the parameters as follows:

$$
a_{11}=1, a_{12}=2, a_{13}=0.5, a_{14}=0.18387 .
$$

The eigenvalues of the Jacobian matrix at the equilibrium $(0.70711,0,0.84251,0)$ point are $\pm 1.3963 i$ and $-0.18387 \pm 1.81914 i$, and the Hopf bifurcation occurs in the system (1). Based on Theorem 3, we get $\mu=-\frac{47.03741 \pi}{11.1704}-\frac{41.82834 \pi}{15.59723}<0$, then the Hopf bifurcation at the equilibrium point of system (1) is non-degenerate, and the bifurcating periodic solution is stable. Because of the symmetry of the system (1), the same type of Hopf bifurcation occurs at the equilibrium $(-0.70711,0,-0.84251,0)$. The phase diagrams in the $p$ - $u$ plane and the $q-v$ plane of system (1) with initial values $(0.7,0,-0.01,0)$ are given in Figures 1 and 2, respectively. It is shown that the system has a stable limit cycle around the equilibrium point. Moreover, the eigenvalues of the Jacobian matrix at the equilibria $(0,0,0,0)$, $(0,0,0.70711,0)$, and $(0,0,-0.70711,0)$ are:

$$
1.15099,0.68014,1.0994 \pm 0.261998 i \text {, and } 0.95,-1.05018,-0.13378 \pm 1.40954 i \text {, }
$$


and $0.95,-1.05018,-0.13378 \pm 1.40954 i$, respectively. Thus, $(0,0,0,0)$ is a stable-focus point with a four-dimensional unstable manifold, and $(0,0,0.70711,0),(0,0,-0.70711,0)$ are all stable-focus points with a three-dimensional stable manifold and a one-dimensional unstable manifold.

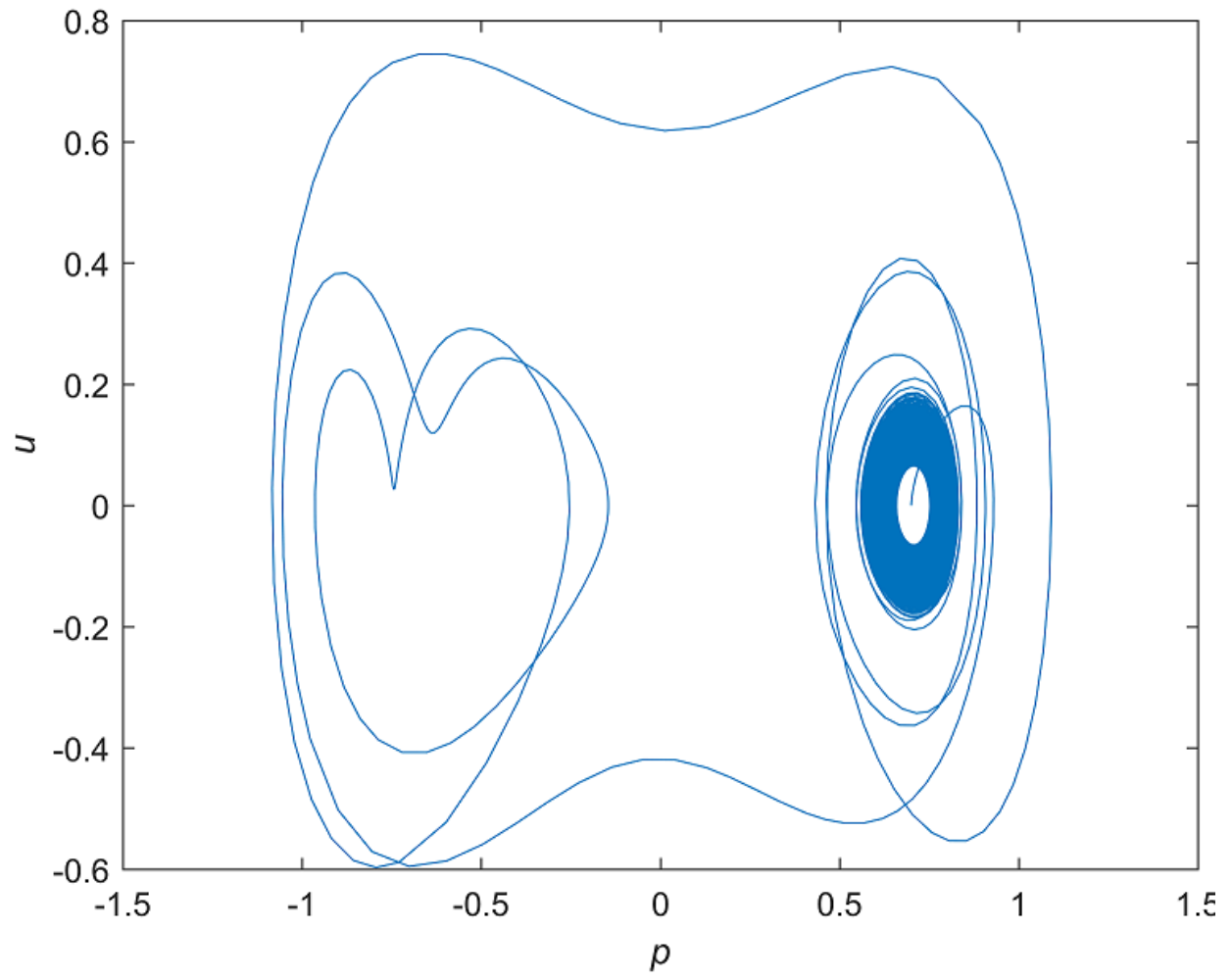

Figure 1. The phase diagram in the $p-u$ plane.

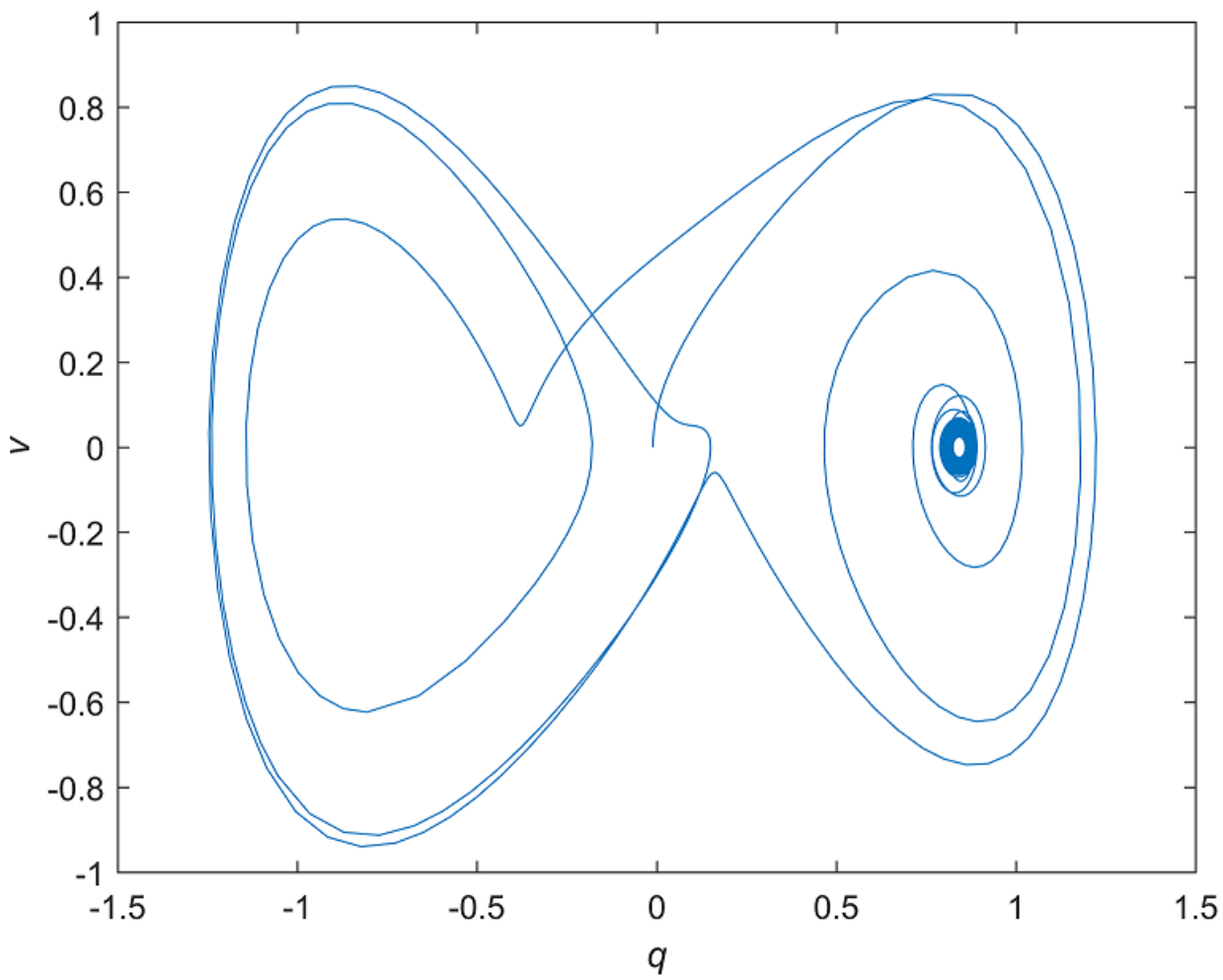

Figure 2. The phase diagram in the $q-v$ plane.

Next, we assume the parameters as follows: 


$$
a_{11}=1, a_{12}=2, a_{13}=0.2, a_{14}=0.01 .
$$

In this case, the system (1) has nine equilibrium points. Table 1 indicates the eigenvalues of the corresponding Jacobian matrix and the equilibria type and shows the unstable manifold and stable manifold at the equilibrium points of the particle motion system.

Table 1. The eigenvalues of the corresponding Jacobian matrix and the equilibria type.

\begin{tabular}{llc}
\hline Equilibrium Points & Eigenvalues of the Jacobian Matrix & Equilibria Type \\
\hline$(0,0,0,0)$ & $1.09467,0.89543,-1.005 \pm 0.10037 i$ & saddle-focus point \\
$(0,0,0.70711,0)$ & $1.00164,-0.9983,-0.01167 \pm 1.41421 i$ & saddle-focus point \\
$(0,0,-0.70711,0)$ & $1.00164,-0.9983,-0.01167 \pm 1.41421 i$ & saddle-focus point \\
$(-0.70711,0,0.14789,0)$ & $0.93402,-0.93008,-0.01197 \pm 1.41421 i$ & saddle-focus point \\
$(0.70711,0,-0.14789,0)$ & $0.93402,-0.93008,-0.01197 \pm 1.41421 i$ & saddle-focus point \\
$(-0.70711,0,0.62147,0)$ & $-0.03366 \pm 1.41718 i, 0.02366 \pm 1.14477 i$ & focus point \\
$(0.70711,0,-0.62147,0)$ & $-0.03366 \pm 1.41718 i, 0.02366 \pm 1.14477 i$ & focus point \\
$(0.70711,0,0.76936,0)$ & $-0.03366 \pm 1.41718 i, 0.023657 \pm 1.14477 i$ & focus point \\
$(-0.70711,0,-0.76936,0)$ & $-0.03366 \pm 1.41718 i, 0.023657 \pm 1.14477 i$ & focus point \\
\hline
\end{tabular}

It has been long supposed that the existence of chaotic behavior in the microscopic motions is responsible for their equilibrium and non-equilibrium properties [18], and the increase of the equilibrium points or multi-equilibrium points may lead to abundant dynamic behaviors of the system [8-10]. From the above results, it is shown that the system (1) has multi-equilibrium points with a stable manifold and an unstable manifold. Therefore, the particle motion system has rich dynamic behaviors. The Lyapunov exponents are $0.01,0.00,-0.01$, and -0.02 by using the method in [19]; thus, the system (1) is chaotic. In addition, the chaotic phenomena can also be reflected by the Poincare maps [20]. Therefore, the chaotic attractor in the $p-q$ plane of System (1) with parameters $a_{11}=1, a_{12}=2, a_{13}=0.2, a_{14}=0.01$ and initial values $(0.7,0,-0.01,0)$ is shown in Figure $3 a$, and the Poincaré mapping on the section hyperplane $u=0$ is given in Figure $3 \mathrm{~b}$. The chaotic attractor in the $u-v$ plane of System (1) with parameters $a_{11}=1, a_{12}=2, a_{13}=0.2, a_{14}=0.01$ and initial values $(0.7,0,-0.01,0)$ is shown in Figure $4 \mathrm{a}$, and the Poincare mapping on the section hyperplane $p=0$ is given in Figure $4 \mathrm{~b}$. Here, the Runge-Kutta method of order four is employed with the time step of 0.001 from $t=0$ to $t=300$. This shows that the particle motion trajectories and the velocities of the particle in both directions are complex and the particle motion chaotic. Hence, the particle motion system shows chaotic behavior. To further illustrate the strange attractors of System (1), Figures 5 and 6 show the chaos phase portrait of $p-u$ and $q-v$ and the corresponding Poincaré map by taking the same parameters and initial values as Figure 3.
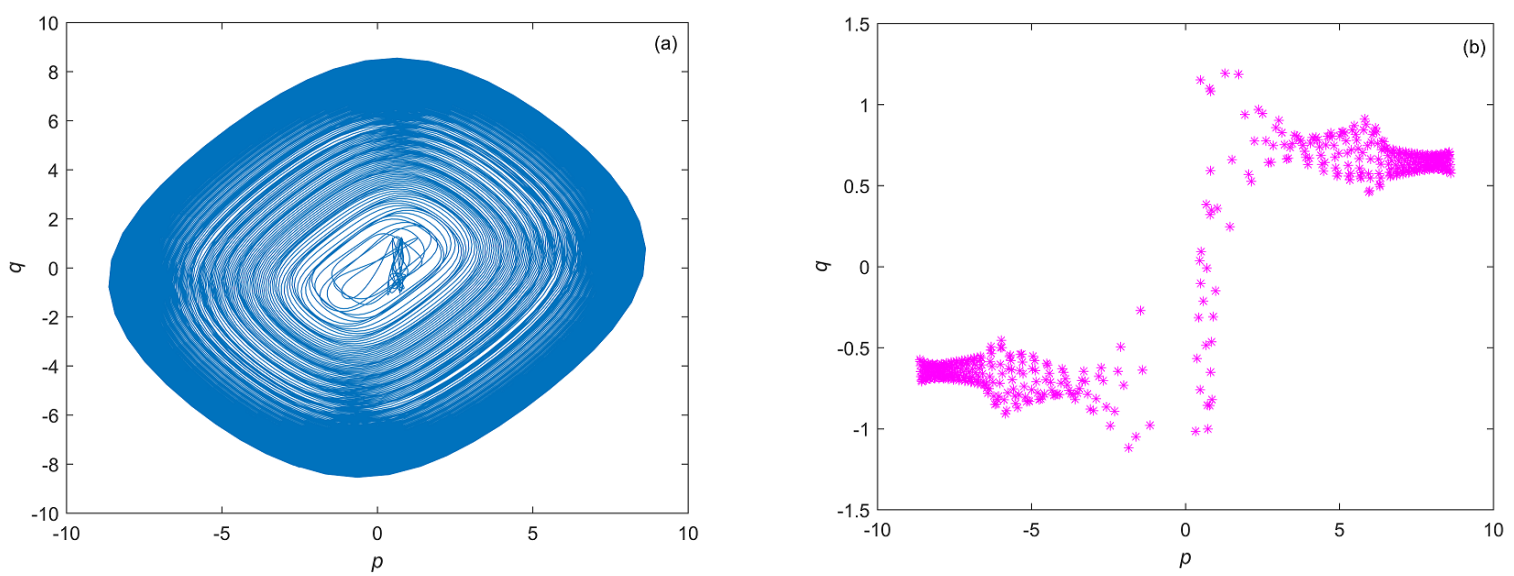

Figure 3. (a) The chaotic attractor in the $p$ - $q$ plane; (b) The Poincaré mapping on the section $u=0$. 

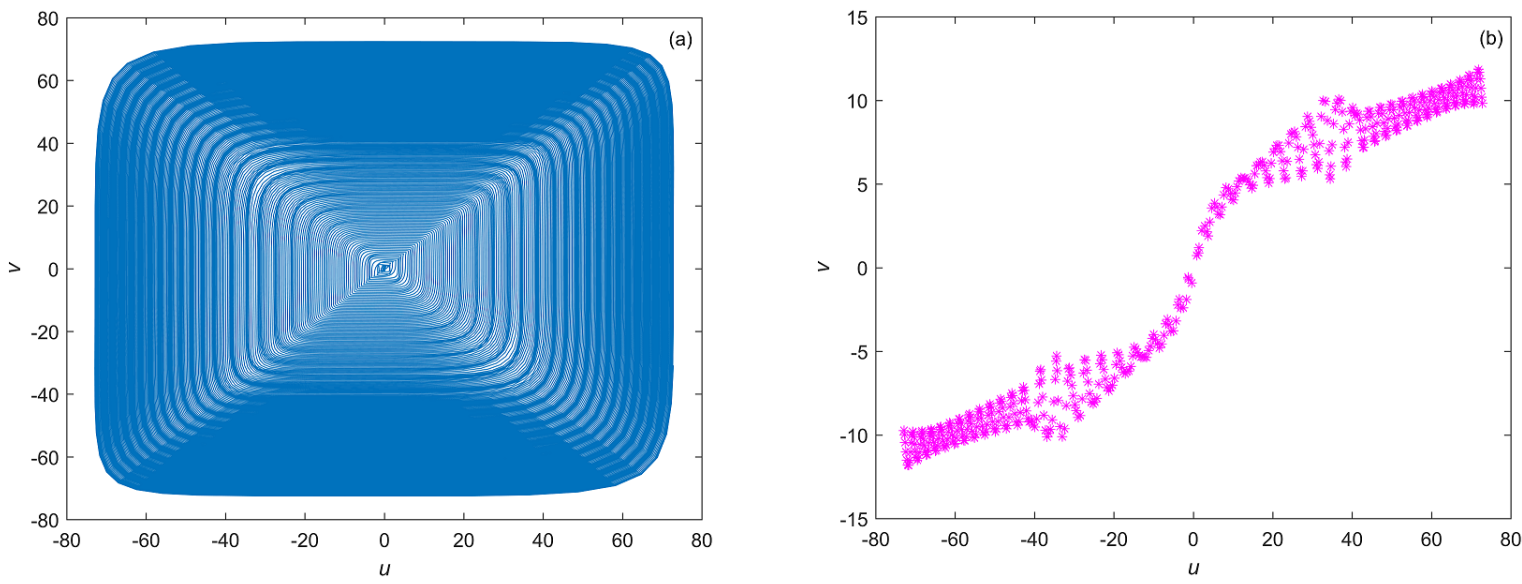

Figure 4. (a) The chaotic attractor in the $u$-v plane; (b) The Poincaré mapping on the section $p=0$.

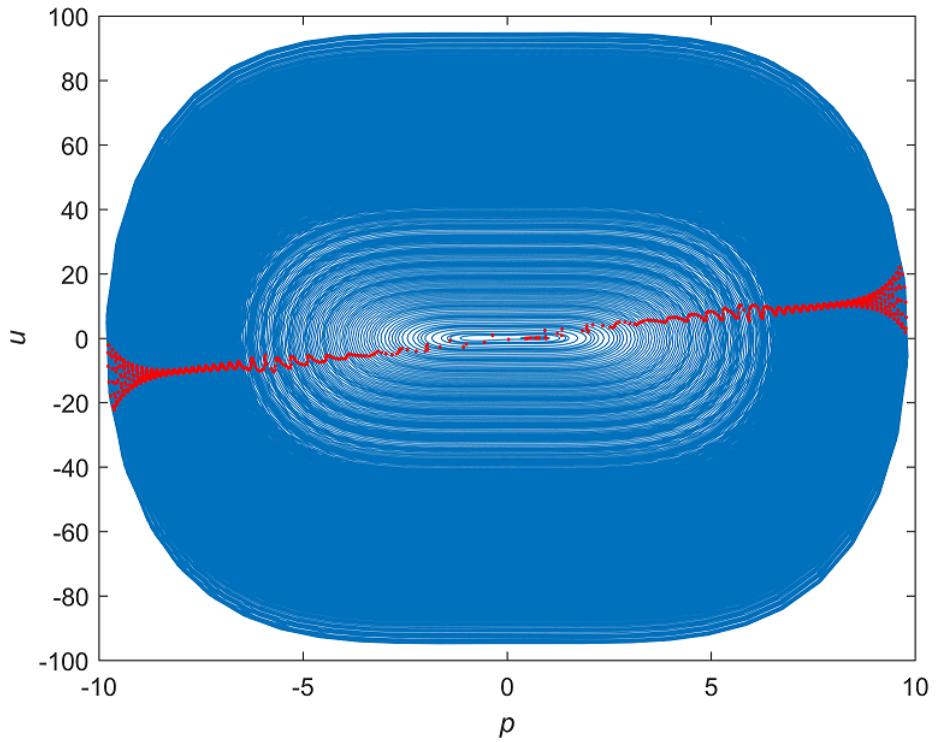

Figure 5. $p-u$ phase portrait and the corresponding Poincaré map (red points) on the section $q=0$.

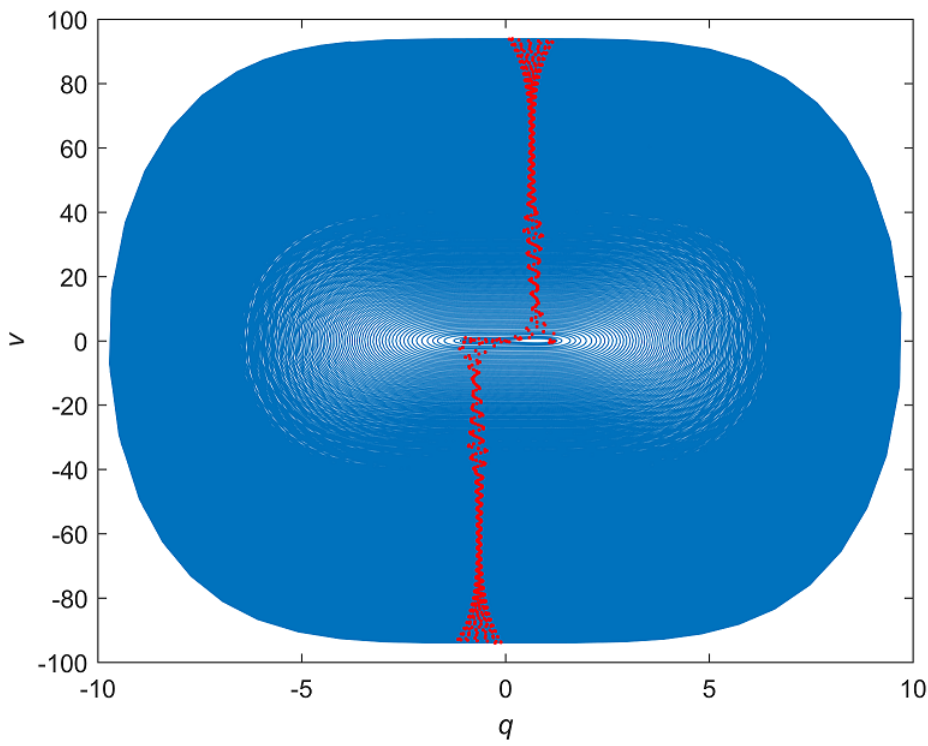

Figure 6. $q-v$ phase portrait and the corresponding Poincaré map (red points) on the section $u=0$. 


\section{Conclusions}

In this paper, a particle motion model is formulated by introducing external forces. The dynamic behaviors of the system are investigated, including the symmetry, the existence and uniqueness of the solution, and the continuous dependence on initial conditions. The range of the parameter where the solution of the system shows continuous dependence on initial conditions can be determined from Theorems 1 and 2. Consequently, the range of parameter values of the system where the system does not exhibit chaotic behavior can be determined in theory. The results provide great help in controlling the dynamic behavior of the particle motion system. By using the center manifold theorem and simulations, the Hopf bifurcations at the equilibrium and chaotic behavior are studied. This illustrates that the particle motion system has rich dynamic phenomena and also indicates the influence of the external force on the particle motion trajectories. Compared to [21,22], different results are obtained, such as Theorems 1 and 2, and the dynamic behaviors of the particle motion system are investigated by applying different methods such as the method in [17] and the Poincare section. These results are helpful for further understanding the state of particle motion under external force. How to effectively control the chaotic behavior and bifurcation phenomena of particle motion will be our next research direction.

Author Contributions: All authors have equally contributed to this work. All authors revised and edited the final version of the manuscript.

Funding: The project was supported by the Youth Science Foundations of Education Department of Hebei Province (No. QN2016265), the Hebei Special Foundation "333 talent project" (No. A2016001123), and the Scientific Research Funds of Hebei Institute of Architecture and Civil Engineering (Nos. 2016XJJQN03, 2016XJJYB05).

Acknowledgments: The authors would like to thank the editor and referees for their positive and constructive comments, which are all valuable and very helpful for improving this paper.

Conflicts of Interest: The authors declare no conflict of interest.

\section{Appendix A}

(1) Expressions of $F_{i}\left(y_{1}, y_{2}, y_{3}, y_{4}\right)(i=1,2,3,4)$ in Section 2.5:

$$
\begin{aligned}
& F_{1}\left(y_{1}, y_{2}, y_{3}, y_{4}\right)=b_{12}\left[-a_{12}\left(\frac{\left(-\alpha^{2}-M\right) y_{1}}{a_{13}}-\frac{a_{14} \alpha y_{2}}{a_{13}}+\beta_{22} y_{3}-\beta_{21} y_{4}\right)^{3}-3 a_{12}\left(\frac{\left(-\alpha^{2}-M\right) y_{1}}{a_{13}}-\frac{a_{14} \alpha y_{2}}{a_{13}}\right.\right. \\
& \left.\left.\quad+\beta_{22} y_{3}-\beta_{21} y_{4}\right)^{2} \sqrt{\frac{a_{11}}{a_{12}}}\right]+b_{14}\left[-a_{12}\left(y_{1}+y_{3}\right)^{3}-3 a_{12}\left(y_{1}+y_{3}\right)^{2}\left(\frac{\sqrt[3]{\theta_{1}}}{6 a_{12}}+\frac{2 a_{11}}{\sqrt[3]{\theta_{1}}}\right)\right], \\
& F_{2}\left(y_{1}, y_{2}, y_{3}, y_{4}\right)=b_{22}\left[-a_{12}\left(\frac{\left(-\alpha^{2}-M\right) y_{1}}{a_{13}}-\frac{a_{14} \alpha y_{2}}{a_{13}}+\beta_{22} y_{3}-\beta_{21} y_{4}\right)^{3}-3 a_{12}\left(\frac{\left(-\alpha^{2}-M\right) y_{1}}{a_{13}}-\frac{a_{14} \alpha y_{2}}{a_{13}}\right.\right. \\
& \left.\left.\quad+\beta_{22} y_{3}-\beta_{21} y_{4}\right)^{2} \sqrt{\frac{a_{11}}{a_{12}}}\right]+b_{24}\left[-a_{12}\left(y_{1}+y_{3}\right)^{3}-3 a_{12}\left(y_{1}+y_{3}\right)^{2}\left(\frac{\sqrt[3]{\theta_{1}}}{6 a_{12}}+\frac{2 a_{11}}{\sqrt[3]{\theta_{1}}}\right)\right], \\
& F_{3}\left(y_{1}, y_{2}, y_{3}, y_{4}\right)=b_{32}\left[-a_{12}\left(\frac{\left(-\alpha^{2}-M\right) y_{1}}{a_{13}}-\frac{a_{14} \alpha y_{2}}{a_{13}}+\beta_{22} y_{3}-\beta_{21} y_{4}\right)^{3}-3 a_{12}\left(\frac{\left(-\alpha^{2}-M\right) y_{1}}{a_{13}}-\frac{a_{14} \alpha y_{2}}{a_{13}}\right.\right. \\
& \left.\left.\quad+\beta_{22} y_{3}-\beta_{21} y_{4}\right)^{2} \sqrt{\frac{a_{11}}{a_{12}}}\right]+b_{34}\left[-a_{12}\left(y_{1}+y_{3}\right)^{3}-3 a_{12}\left(y_{1}+y_{3}\right)^{2}\left(\frac{\sqrt[3]{\theta_{1}}}{6 a_{12}}+\frac{2 a_{11}}{\sqrt[3]{\theta_{1}}}\right)\right], \\
& F_{4}\left(y_{1}, y_{2}, y_{3}, y_{4}\right)=b_{42}\left[-a_{12}\left(\frac{\left(-\alpha^{2}-M\right) y_{1}}{a_{13}}-\frac{a_{14} \alpha y_{2}}{a_{13}}+\beta_{22} y_{3}-\beta_{21} y_{4}\right)^{3}-3 a_{12}\left(\frac{\left(-\alpha^{2}-M\right) y_{1}}{a_{13}}-\frac{a_{14} \alpha y_{2}}{a_{13}}\right.\right. \\
& \left.\left.\quad+\beta_{22} y_{3}-\beta_{21} y_{4}\right)^{2} \sqrt{\frac{a_{11}}{a_{12}}}\right]+b_{44}\left[-a_{12}\left(y_{1}+y_{3}\right)^{3}-3 a_{12}\left(y_{1}+y_{3}\right)^{2}\left(\frac{\sqrt[3]{\theta_{1}}}{6 a_{12}}+\frac{2 a_{11}}{\sqrt[3]{\theta_{1}}}\right)\right],
\end{aligned}
$$

where:

$$
\begin{aligned}
& b_{11}=-\frac{1}{\Delta_{1}}\left[\left(\alpha a_{13} \beta_{23}+\alpha\left(\alpha^{2}+M\right) \beta_{25}\right) a_{13}\right], b_{12}=\frac{1}{\Delta_{1}}\left[\left(a_{13} \beta_{21}-a_{14} \beta_{25}\right) \alpha a_{13}\right], \\
& b_{13}=\frac{1}{\Delta_{1}}\left[a _ { 1 3 } \left(-\alpha a_{13} \beta_{21} \beta_{24}+\alpha a_{13} \beta_{22} \beta_{23}+\alpha a_{14} \beta_{23}+\alpha a_{14} \beta_{24} \beta_{25}+\alpha\left(\alpha^{2}+M\right) a_{14} \beta_{21}\right.\right. \\
& \left.\left.+\alpha\left(\alpha^{2}+M\right) \beta_{22} \beta_{25}\right)\right], b_{14}=\frac{1}{\Delta_{1}}\left[\left(\alpha a_{14} \beta_{23}+\alpha\left(\alpha^{2}+M\right) \beta_{21}\right) a_{13}\right], \\
& b_{21}=-\frac{1}{\Delta_{1}}\left[\left(\alpha^{2} a_{14} \beta_{25}+a_{13} a_{14} \beta_{23}+a_{13} \beta_{24} \beta_{25}\right) a_{13}\right], \\
& b_{22}=\frac{1}{\Delta_{1}}\left[\left(\alpha^{2} \beta_{25}+a_{13} a_{14} \beta_{21}+a_{13} \beta_{22} \beta_{25}+M \beta_{25}\right) a_{13}\right], \\
& b_{23}=-\frac{1}{\Delta_{1}}\left[\left(-\alpha^{2} a_{14}{ }^{2} \beta_{21}-\alpha^{2} a_{14} \beta_{22} \beta_{25}+\alpha^{2} a_{14} \beta_{23}+\alpha^{2} \beta_{24} \beta_{25}+M a_{14} \beta_{23}+M \beta_{24} \beta_{25}\right) a_{13}\right],
\end{aligned}
$$




$$
\begin{aligned}
& b_{24}=-\frac{1}{\Delta_{1}}\left[\left(-\alpha^{2} a_{14} \beta_{21}+\alpha^{2} \beta_{23}-a_{13} \beta_{21} \beta_{24}+a_{13} \beta_{22} \beta_{23}+M \beta_{23}\right) a_{13}\right], \\
& b_{31}=\frac{1}{\Delta_{1}}\left[\left(\alpha a_{13} \beta_{23}+\alpha\left(\alpha^{2}+M\right) \beta_{25}\right) a_{13}\right], b_{32}=-\frac{1}{\Delta_{1}}\left[\left(a_{13} \beta_{21}-a_{14} \beta_{25}\right) \alpha a_{13}\right], \\
& b_{33}=\frac{1}{\Delta_{1}}\left[-\alpha^{3} a_{13} a_{14} \beta_{21}+\alpha^{3} a_{14}{ }^{2} \beta_{25}+\alpha^{3} a_{13} \beta_{23}+M \alpha a_{13} \beta_{23}+\alpha\left(\alpha^{2}+M\right) \alpha^{2} \beta_{25}+M \alpha\left(\alpha^{2}+M\right) \beta_{25}\right], \\
& b_{34}=-\frac{1}{\Delta_{1}}\left[\left(\alpha a_{14} \beta_{23}+\alpha\left(\alpha^{2}+M\right) \beta_{21}\right) a_{13}\right], b_{41}=-\frac{1}{\Delta_{1}}\left[\left(-\alpha^{3} a_{14}-\alpha a_{13} \beta_{24}+\alpha\left(\alpha^{2}+M\right) a_{14}\right) a_{13}\right], \\
& b_{42}=-\frac{1}{\Delta_{1}}\left[\left(\alpha^{2}+a_{13} \beta_{22}+a_{14}{ }^{2}+M\right) \alpha a_{13}\right], \\
& b_{43}=-\frac{1}{\Delta_{1}}\left[\alpha^{3} a_{13} a_{14} \beta_{22}+\alpha^{3} a_{14}{ }^{3}-\alpha^{3} a_{13} \beta_{24}-M \alpha a_{13} \beta_{24}+\alpha\left(\alpha^{2}+M\right) \alpha^{2} a_{14}+M \alpha\left(\alpha^{2}+M\right) a_{14}\right], \\
& b_{44}=-\frac{1}{\Delta_{1}}\left[\alpha^{3} a_{14}{ }^{2}+\alpha a_{13} a_{14} \beta_{24}+\alpha\left(\alpha^{2}+M\right) \alpha^{2}+\alpha\left(\alpha^{2}+M\right) a_{13} \beta_{22}+M \alpha\left(\alpha^{2}+M\right)\right] . \\
& \Delta_{1}=-\alpha^{3} a_{13} a_{14} \beta_{21}+\alpha^{3} a_{14}{ }^{2} \beta_{25}+\alpha^{3} a_{13} \beta_{23}-\alpha a_{13}{ }^{2} \beta_{21} \beta_{24}+\alpha a_{13} \beta_{22} \beta_{23}+\alpha a_{13} a_{14}^{2} \beta_{23} \\
& \quad+\alpha a_{13} a_{14} \beta_{24} \beta_{25}+M \alpha a_{13} \beta_{23}+\alpha\left(\alpha^{2}+M\right) \alpha^{2} \beta_{25}+\alpha\left(\alpha^{2}+M\right) a_{13} a_{14} \beta_{21} \\
& \quad+\alpha\left(\alpha^{2}+M\right) a_{13} \beta_{22} \beta_{25}+M \alpha\left(\alpha^{2}+M\right) \beta_{25}
\end{aligned}
$$

(2) Expressions of $h_{3 j}, h_{4 j}(j=1,2,3)$ in Section 2.5:

$$
h_{31}=\frac{1}{\Delta_{2}}\left|\begin{array}{cccccc}
0 & -\alpha & 0 & -\frac{\sqrt{-\Delta_{0}}}{2} & 0 & 0 \\
0 & 0 & -2 \alpha & 0 & -\frac{\sqrt{-\Delta_{0}}}{2} & 0 \\
0 & \alpha & 0 & 0 & 0 & -\frac{\sqrt{-\Delta_{0}}}{2} \\
\delta_{21} & 0 & 0 & -a_{14} & -\alpha & 0 \\
\delta_{22} & \frac{\sqrt{-\Delta_{0}}}{2} & 0 & 2 \alpha & -a_{14} & -2 \alpha \\
\delta_{23} & 0 & \frac{\sqrt{-\Delta_{0}}}{2} & 0 & \alpha & -a_{14} \\
0 & 0 & 0 & -\frac{\sqrt{-\Delta_{0}}}{2} & 0 & 0 \\
2 \alpha & 0 & -2 \alpha & 0 & -\frac{\sqrt{-\Delta_{0}}}{2} & 0 \\
0 & 0 & 0 & 0 & 0 & -\frac{\sqrt{-\Delta_{0}}}{2} \\
\frac{\sqrt{-\Delta_{0}}}{2} & \delta_{21} & 0 & -a_{14} & -\alpha & 0 \\
0 & \delta_{22} & 0 & 2 \alpha & -a_{14} & -2 \alpha \\
0 & \delta_{23} & \frac{\sqrt{-\Delta_{0}}}{2} & 0 & \alpha & -a_{14} \\
0 & -\alpha & 0 & -\frac{\sqrt{-\Delta_{0}}}{2} & 0 & 0 \\
2 \alpha & 0 & 0 & 0 & -\frac{\sqrt{-\Delta_{0}}}{2} & 0 \\
0 & \alpha & 0 & 0 & 0 & -\frac{\sqrt{-\Delta_{0}}}{2} \\
\frac{\sqrt{-\Delta_{0}}}{2} & 0 & \delta_{21} & -a_{14} & -\alpha & 0 \\
0 & \frac{\sqrt{-\Delta_{0}}}{2} & \delta_{22} & 2 \alpha & -a_{14} & -2 \alpha \\
0 & 0 & \delta_{23} & 0 & \alpha & -a_{14}
\end{array}\right|,
$$




$$
h_{41}=\frac{1}{\Delta_{2}}\left|\begin{array}{cccccc}
0 & -\alpha & 0 & 0 & 0 & 0 \\
2 \alpha & 0 & -2 \alpha & 0 & -\frac{\sqrt{-\Delta_{0}}}{2} & 0 \\
0 & \alpha & 0 & 0 & 0 & -\frac{\sqrt{-\Delta_{0}}}{2} \\
\frac{\sqrt{-\Delta_{0}}}{2} & 0 & 0 & \delta_{21} & -\alpha & 0 \\
0 & \frac{\sqrt{-\Delta_{0}}}{2} & 0 & \delta_{22} & -a_{14} & -2 \alpha \\
0 & 0 & \frac{\sqrt{-\Delta_{0}}}{2} & \delta_{23} & \alpha & -a_{14}
\end{array}\right|,
$$

where:

$$
\begin{aligned}
& \delta_{21}=-\frac{3 b_{42} a_{12}\left(-\alpha^{2}-M\right)^{2}}{a_{13} 3^{2}} \sqrt{\frac{a_{11}}{a_{12}}}-3 b_{44} a_{12}\left(\frac{\sqrt[3]{\theta_{1}}}{6 a_{12}}+\frac{2 a_{11}}{\sqrt[3]{\theta_{1}}}\right), \\
& \delta_{22}=\frac{6 b_{42} a_{12} a_{14} \alpha\left(-\alpha^{2}-M\right)}{a_{13}{ }^{2}} \sqrt{\frac{a_{11}}{a_{12}}} \\
& \delta_{23}=-\frac{3 b_{42} a_{12} a_{14} \alpha^{2}}{a_{13}{ }^{2}} \sqrt{\frac{a_{11}}{a_{12}}} \\
& \Delta_{2}=-4 \Delta_{0} \alpha^{4}-\Delta_{0} a_{14}{ }^{2} \alpha^{2}-\frac{1}{2} \alpha^{2} \Delta_{0}^{2}-\frac{\Delta_{0}^{3}}{64} .
\end{aligned}
$$

(3) Expressions of $G_{1}\left(y_{1}, y_{2}\right)$ and $G_{2}\left(y_{1}, y_{2}\right)$ in Section 2.5:

$$
\begin{aligned}
& G_{1}\left(y_{1}, y_{2}\right)=\left[b_{12}\left(-\frac{a_{12}\left(-\alpha^{2}-M\right)^{3}}{a_{13} 3^{3}}-\frac{6 a_{12}\left(-\alpha^{2}-M\right)\left(-\beta_{21} h_{41}+\beta_{22} h_{31}\right)}{a_{13}} \sqrt{\frac{a_{11}}{a_{12}}}\right)+b_{14}\left(-a_{12}-6 a_{12} h_{31}\left(\frac{\sqrt[3]{\theta_{1}}}{6 a_{12}}\right.\right.\right. \\
& \left.\left.\left.\quad+\frac{2 a_{11}}{\sqrt[3]{\theta_{1}}}\right)\right)\right] y_{1}^{3}+\left[b _ { 1 2 } \left(\frac{3 a_{12} a_{14} \alpha\left(-\alpha^{2}-M\right)^{2}}{a_{13}{ }^{3}}-3 a_{12}\left(-\frac{2 a_{14} \alpha\left(-\beta_{21} h_{41}+\beta_{22} h_{31}\right)}{a_{13}}+\frac{2\left(-\alpha^{2}-M\right)\left(-\beta_{21} h_{42}+\beta_{22} h_{32}\right)}{a_{13}}\right)\right.\right. \\
& \left.\left.\quad \times \sqrt{\frac{a_{11}}{a_{12}}}\right)-6 b_{14} a_{12} h_{32}\left(\frac{\sqrt[3]{\theta_{1}}}{6 a_{12}}+\frac{2 a_{11}}{\sqrt[3]{\theta_{1}}}\right)\right] y_{1}^{2} y_{2}+\left[b _ { 1 2 } \left(-\frac{3 a_{12} a_{14} \alpha^{2}\left(-\alpha^{2}-M\right)}{a_{13} 3^{3}}-\left(-\frac{6 a_{12} a_{14} \alpha\left(-\beta_{21} h_{42}+\beta_{22} h_{32}\right)}{a_{13}}\right.\right.\right. \\
& \left.\left.\left.\quad+\frac{a_{12}\left(-\beta_{21} h_{43}+\beta_{22} h_{33}\right)\left(-\alpha^{2}-M\right)}{a_{13}}\right) \sqrt{\frac{a_{11}}{a_{12}}}\right)-6 b_{14} a_{12} h_{33}\left(\frac{\sqrt[3]{\theta_{1}}}{6 a_{12}}+\frac{2 a_{11}}{\sqrt[3]{\theta_{1}}}\right)\right] y_{1} y_{2}^{2}+\left[b _ { 1 2 } \left(\frac{a_{12} a_{14}^{3} \alpha^{3}}{a_{13}^{3}}\right.\right. \\
& \left.\left.\quad+\frac{6 a_{12} a_{14} \alpha\left(-\beta_{21} h_{43}+\beta_{22} h_{33}\right)}{a_{13}} \sqrt{\frac{a_{11}}{a_{12}}}\right)\right] y_{2}^{3}+o\left(\left\|y_{1}, y_{2}\right\|^{4}\right), \\
& G_{2}\left(y_{1}, y_{2}\right)=\left[b_{22}\left(-\frac{a_{12}\left(-\alpha^{2}-M\right)^{3}}{a_{13}}-\frac{6 a_{12}\left(-\alpha^{2}-M\right)\left(-\beta_{21} h_{41}+\beta_{22} h_{31}\right)}{a_{13}} \sqrt{\frac{a_{11}}{a_{12}}}\right)+b_{24}\left(-a_{12}-6 a_{12} h_{31}\left(\frac{\sqrt[3]{\theta_{1}}}{6 a_{12}}\right.\right.\right. \\
& \left.\left.\quad+\frac{2 a_{11}}{\sqrt[3]{\theta_{1}}}\right)\right] y_{1}^{3}+\left[b _ { 2 2 } \left(\frac{3 a_{12} a_{14} \alpha\left(-\alpha^{2}-M\right)^{2}}{a_{13}}-3 a_{12}\left(-\frac{2 a_{14} \alpha\left(-\beta_{21} h_{41}+\beta_{22} h_{31}\right)}{a_{13}}+\frac{2\left(-\alpha^{2}-M\right)\left(-\beta_{21} h_{42}+\beta_{22} h_{32}\right)}{a_{13}}\right)\right.\right.
\end{aligned}
$$




$$
\begin{aligned}
& \left.\left.\times \sqrt{\frac{a_{11}}{a_{12}}}\right)-6 b_{24} a_{12} h_{32}\left(\frac{\sqrt[3]{\theta_{1}}}{6 a_{12}}+\frac{2 a_{11}}{\sqrt[3]{\theta_{1}}}\right)\right] y_{1}^{2} y_{2}+\left[b _ { 2 2 } \left(-\frac{3 a_{12} a_{14}^{2} \alpha^{2}\left(-\alpha^{2}-M\right)}{a_{13}{ }^{3}}-\left(-\frac{6 a_{12} a_{14} \alpha\left(-\beta_{21} h_{42}+\beta_{22} h_{32}\right)}{a_{13}}\right.\right.\right. \\
& \left.\left.\left.+\frac{a_{12}\left(-\beta_{21} h_{43}+\beta_{22} h_{33}\right)\left(-\alpha^{2}-M\right)}{a_{13}}\right) \sqrt{\frac{a_{11}}{a_{12}}}\right)-6 b_{24} a_{12} h_{33}\left(\frac{\sqrt[3]{\theta_{1}}}{6 a_{12}}+\frac{2 a_{11}}{\sqrt[3]{\theta_{1}}}\right)\right] y_{1} y_{2}^{2}+\left[b _ { 2 2 } \left(\frac{a_{12} a_{14} \alpha^{3}}{a_{13}{ }^{3}}\right.\right. \\
& \left.\left.+\frac{6 a_{12} a_{14} \alpha\left(-\beta_{21} h_{43}+\beta_{22} h_{33}\right)}{a_{13}} \sqrt{\frac{a_{11}}{a_{12}}}\right)\right] y_{2}^{3}+o\left(\left\|y_{1}, y_{2}\right\|^{4}\right) .
\end{aligned}
$$

(4) Expressions of $\mu_{1}$ and $\mu_{2}$ in Section 2.5:

$$
\begin{aligned}
& \mu_{1}=b_{12}\left(-\frac{6 a_{12}\left(-\alpha^{2}-M\right)^{3}}{a_{13}{ }^{3}}-\frac{18 a_{12}\left(-\alpha^{2}-M\right)\left(-2 \beta_{21} h_{41}+2 \beta_{22} h_{31}\right)}{a_{13}} \sqrt{\frac{a_{11}}{a_{12}}}\right)+b_{14}\left(-6 a_{12}-36 a_{12}\left(\frac{\sqrt[3]{\theta_{1}}}{6 a_{12}}\right.\right. \\
& \left.\left.+\frac{2 a_{11}}{\sqrt[3]{\theta_{1}}}\right) h_{31}\right)+b_{12}\left(-\frac{6 a_{12} a_{14}{ }^{2} \alpha^{2}\left(-\alpha^{2}-M\right)}{a_{13}{ }^{3}}-\frac{6 a_{12}\left(-2 \beta_{21} h_{43}+2 \beta_{22} h_{33}\right)\left(-\alpha^{2}-M\right)}{a_{13}} \sqrt{\frac{a_{11}}{a_{12}}}\right. \\
& \left.+\frac{12 a_{12} a_{14} \alpha\left(-\beta_{21} h_{42}+\beta_{22} h_{32}\right)}{a_{13}} \sqrt{\frac{a_{11}}{a_{12}}}\right)-12 b_{14} a_{12} h_{33}\left(\frac{\sqrt[3]{\theta_{1}}}{6 a_{12}}+\frac{2 a_{11}}{\sqrt[3]{\theta_{1}}}\right)+b_{22}\left(\frac{6 a_{12} a_{14} \alpha\left(-\alpha^{2}-M\right)^{2}}{a_{13}{ }^{3}}\right. \\
& \left.-\frac{12 a_{12}\left(-\alpha^{2}-M\right)\left(-\beta_{21} h_{42}+\beta_{22} h_{32}\right)}{a_{13}} \sqrt{\frac{a_{11}}{a_{12}}}+\frac{6 a_{12} a_{14} \alpha\left(-2 \beta_{21} h_{41}+2 \beta_{22} h_{31}\right)}{a_{13}} \sqrt{\frac{a_{11}}{a_{12}}}\right)-12 b_{24} a_{12}\left(\frac{\sqrt[3]{\theta_{1}}}{6 a_{12}}\right. \\
& \left.+\frac{2 a_{11}}{\sqrt[3]{\theta_{1}}}\right) h_{32}+b_{22}\left(\frac{6 a_{12} a_{14} \alpha^{3}}{a_{13}{ }^{3}}+\frac{18 a_{12} a_{14} \alpha\left(-2 \beta_{21} h_{43}+2 \beta_{22} h_{33}\right)}{a_{13}} \sqrt{\frac{a_{11}}{a_{12}}}\right), \\
& \mu_{2}=\frac{6 b_{12} a_{12} a_{14} \alpha\left(-\alpha^{2}-M\right)}{a_{13}{ }^{2}} \sqrt{\frac{a_{11}}{a_{12}}}\left(-\frac{6 b_{12} a_{12}\left(-\alpha^{2}-M\right)^{2}}{a_{13}{ }^{2}} \sqrt{\frac{a_{11}}{a_{12}}}-6 b_{14} a_{12}\left(\frac{\sqrt[3]{\theta_{1}}}{6 a_{12}}+\frac{2 a_{11}}{\sqrt[3]{\theta_{1}}}\right)-\frac{6 b_{12} a_{12} a_{14} \alpha^{2}}{a_{13}{ }^{2}} \sqrt{\frac{a_{11}}{a_{12}}}\right) \\
& -\frac{6 b_{22} a_{12} a_{14} \alpha\left(-\alpha^{2}-M\right)}{a_{13}{ }^{2}} \sqrt{\frac{a_{11}}{a_{12}}}\left(-\frac{6 b_{22} a_{12}\left(-\alpha^{2}-M\right)^{2}}{a_{13}{ }^{2}} \sqrt{\frac{a_{11}}{a_{12}}}-6 b_{24} a_{12}\left(\frac{\sqrt[3]{\theta_{1}}}{6 a_{12}}+2 \frac{a_{11}}{\sqrt[3]{\theta_{1}}}\right)-\frac{6 b_{22} a_{12} a_{14}{ }^{2} \alpha^{2}}{a_{13}{ }^{2}} \sqrt{\frac{a_{11}}{a_{12}}}\right) \\
& -\left(-\frac{6 b_{12} a_{12}\left(-\alpha^{2}-M\right)^{2}}{a_{13}{ }^{2}} \sqrt{\frac{a_{11}}{a_{12}}}-6 b_{14} a_{12}\left(\frac{\sqrt[3]{\theta_{1}}}{6 a_{12}}+\frac{2 a_{11}}{\sqrt[3]{\theta_{1}}}\right)\right)\left(-\frac{6 b_{22} a_{12}\left(-\alpha^{2}-M\right)^{2}}{a_{13}{ }^{2}} \sqrt{\frac{a_{11}}{a_{12}}}-6 b_{24} a_{12}\left(\frac{\sqrt[3]{\theta_{1}}}{6 a_{12}}+\frac{2 a_{11}}{\sqrt[3]{\theta_{1}}}\right)\right) \\
& +\frac{36 b_{12} a_{12} a_{14} \alpha^{4} a_{11} b_{22}}{a_{13}{ }^{4}} \text {. }
\end{aligned}
$$

\section{References}

1. Abbott, N.L. Colloid Science Collides with Liquid Crystals. Science 2013, 342, 1326-1327. [CrossRef] [PubMed]

2. Chen, C.; Davidson, R.C. Chaotic particle dynamics in free-electron lasers. Phys. Rev. A 1991, 43, 5541. [CrossRef] [PubMed]

3. Hashimoto, K.; Tanahashi, N. Universality in chaos of particle motion near black hole horizon. Phys. Rev. D 2017, 95, 024007. [CrossRef]

4. Sprott, J.C.; Xiong, A. Classifying and quantifying basins of attraction. Chaos 2015, 25, 22-30. [CrossRef] [PubMed]

5. Boeing, G. Visual analysis of nonlinear dynamical systems: Chaos, fractals, self-similarity and the limits of prediction. System 2016, 4, 37-54. [CrossRef]

6. Bradley, E.; Kantz, H. Nonlinear time-series analysis revisited. Chaos 2015, 25, 097610. [CrossRef] [PubMed]

7. Sander, E.; Yorke, J.A. The Many Facets of Chaos. Int. J. Bifurcat. Chaos 2015, 25, 1530011. [CrossRef]

8. Yuan, F.; Wang, G.; Wang, X. Extreme multistability in a memristor-based multi-scroll hyperchaotic system. Chaos 2016, 26, 507-519. [CrossRef]

9. Zhou, P.; Yang, F. Hyperchaos, chaos, and horseshoe in a 4D nonlinear system with an infinite number of equilibrium points. Nonlinear Dyn. 2013, 76, 473-480. [CrossRef]

10. Liu, X.; Shen, X.S.; Zhang, H. Multi-scroll chaotic and hyperchaotic attractors generated from chen system. Int. J. Bifurcat. Chaos 2012, 22, 1250033. [CrossRef]

11. Pine, D.J.; Gollub, J.P.; Brady, J.F.; Leshansky, A.M. Chaos and threshold for irreversibility in sheared suspensions. Nature 2005, 438, 997-1000. [CrossRef] [PubMed]

12. Enkeleida, L.; Petia, M.V. Periodic and chaotic orbits of plane-confined micro-rotors in creeping flows. J. Nonlinear Sci. 2015, 25, 1111-1123.

13. AlZahrani, A.M.; Frolov, V.P.; Shoom, A.A. Critical escape velocity for a charged particle moving around a weakly magnetized Schwarzschild black hole. Phys. Rev. D 2013, 87, 084043. [CrossRef]

14. Kuwana, C.M.; Oliveiraz J.A.; Leonel, E.D. A family of dissipative two-dimensional mappings: Chaotic, regular and steady state dynamics investigation. Phys. A 2014, 395, 458-465. [CrossRef] 
15. Oliveira, D.F.; Leonel, E.D. Parameter space for a dissipative Fermi-Ulam model. New J. Phys. 2011, 13, 123012. [CrossRef]

16. Costa, D.R. A dissipative Fermi-Ulam model under two different kinds of dissipation. Commun. Nonlinear Sci. 2015, 22, 1263-1274. [CrossRef]

17. Carr, J. Applications of Centre Manifold Theory; Springer: New York, NY, USA, 1981.

18. Gaspard, P.; Briggs, M.E.; Francis, M.K.; Sengers, J.V.; Gammon, R.W.; Dorfman, J.R.; Calabrese, R.V. Experimental evidence for microscopic chaos. Nature 1998, 6696, 865-868. [CrossRef]

19. Wolf, A.; Swift, J.B.; Swinney, H.L.; Vastano, J.A. Determining Lyapunov exponents from a time series. Physica D 1985, 16, 285-317. [CrossRef]

20. Awrejcewicz, J.; Olejnik, P. Stick-slip dynamics of a two-degree-of-freedom system. Int. J. Bifurcat. Chaos 2013, 13, 843-861. [CrossRef]

21. Li, J.; Wu, H.; Mei, F. Dynamic analysis for the hyperchaotic system with nonholonomic constraints. Nonlinear Dyn. 2017, 90, 2557-2569. [CrossRef]

22. Chang, C.; Ge, Z. Complete identification of chaos of nonlinear nonholonomic systems. Nonlinear Dyn. 2010, 60, 551-559. [CrossRef]

(C) 2018 by the authors. Licensee MDPI, Basel, Switzerland. This article is an open access article distributed under the terms and conditions of the Creative Commons Attribution (CC BY) license (http://creativecommons.org/licenses/by/4.0/). 